\title{
Passive Deflation Time Constant of 7-Week-Old Rabbits
}

\author{
ERNESTO VALDES, W. ROBERT PACE, PETER RICHARDSON, W, SCOTT JARRIEL, AND \\ THOMAS N. HANSEN \\ Department of Pediatrics, Baylor College of Medicine, Houston, Texas 77030
}

\begin{abstract}
The purpose of our study was to determine the effects of insufflation time $\left(T_{1}\right)$ and of tidal volume $\left(V_{T}\right)$ on the passive deflation time constant $(\tau)$ of juvenile rabbits. We sedated and paralyzed nine 7-wk-0ld New Zealand rabbits (wt $1.2 \pm 0.2 \mathrm{SD} \mathrm{kg}$ ) and placed them on a timecycled, pressure-limited mechanical ventilator. Measurements of $\tau$ and dynamic compliance of the respiratory system were made at nine ventilator settings. $T_{1}$ was adjusted to $0.2,0.4$, and $0.6 \mathrm{~s}$ and $V_{T}$ was adjusted to 10,15 , and $20 \mathrm{~mL}$ so that the effects of $T_{I}$ and $V_{T}$ on $\tau$ could be determined independently. Respiratory system quasi-static and static compliances were also measured and served as a basis for discussing the physiologic explanations for the effects of $T_{1}$ and $V_{T}$ on $\tau$. In general, increases in $T_{1}$ resulted in increases in $\tau$ and increases in $V_{T}$ also resulted in increases in $\tau$. Increasing $T_{1}$ from 0.2 to $0.6 \mathrm{~s}$ plus increasing $V_{1}$ from 10 to $20 \mathrm{~mL}$ resulted in an increase in $\tau$ from $0.220 \pm 0.007$ to $0.282 \pm 0.010 \mathrm{~s}$ (mean \pm SEM) $(p<$ 0.05 , student Newman-Kuels), which is a $28 \%$ increase. At a $V_{\mathrm{r}}$ of $10 \mathrm{~mL}$, dynamic compliance increased from 1.95 \pm 0.10 to $2.14 \pm 0.10 \mathrm{~mL} / \mathrm{cm} \mathrm{H}_{2} \mathrm{O}(p<0.05)$ when $\mathrm{T}_{1}$ was increased from 0.2 to $0.6 \mathrm{~s}$; however, dynamic compliance decreased $19 \%$ as $V_{T}$ was increased from 10 to 20 mL. Quasi-static compliance increased from $2.04 \pm 0.07$ to $2.17 \pm 0.12 \mathrm{~mL} / \mathrm{cm} \mathrm{H} \mathrm{H}_{2} \mathrm{O}(p<0.05)$ as deflation volume was increased from 10 to $20 \mathrm{~mL}$, yet static compliance remained relatively constant at $2.6 \mathrm{~mL} / \mathrm{cm} \mathrm{H}_{2} \mathrm{O}$ in the volume range of 10 to $20 \mathrm{~mL}$. We conclude that both $T_{1}$ and $V_{T}$ affect $\tau$ in the small, healthy rabbit and that neither the viscoelastic properties of the lung nor parallel modeling with a distribution of time constants can fully explain our observations. (Pediatr Res 28: 75-78, 1990)
\end{abstract}

\section{Abbreviations}

$T_{\text {Is }}$ insufflation time

$V_{T}$, tidal volume

$\tau$, passive deflation time constant

$C_{d y m}$ dynamic compliance

$\mathrm{C}_{\text {q5 }}$, quasi-state compliance

$\mathrm{C}_{\text {st, }}$ static compliance

Passive deflation time constants are used to characterize the mechanics of the respiratory system and are clinically useful for predicting the time required for a complete exhalation during mechanical ventilation. Shannon (1) suggests that $\tau$, arterial $\mathrm{PCO}_{2}$, alveolar $\mathrm{PCO}_{2}$, and functional residual capacity provide the

Received November 10, 1989, accepted March 19, 1990.

Correspondence: Peter Richardson, Ph.D., Department of Pediatrics, Baylor College of Medicine, One Baylor Plaza, Houston, TX 77030.

Supported by Grant 6-525 from The March of Dimes Birth Defects Foundation. minimum information needed for rational management of infants and children on mechanical ventilation. Because both respiratory gas flow and volume are dependent on $\tau$, to provide optimal gas exchange it is necessary to match the mechanical breathing device to the mechanical properties of the respiratory system.

Recently, experiments in healthy rabbits demonstrated that $\tau$ is influenced by $T_{1}(2)$. This observation could be explained by the visoelastic properties of the respiratory system (3) or by a distribution of passive exhalation time constants (4); presently, there are no methods available for distinguishing if one of the two phenomena dominates.

The purpose of our study was to investigate the effects of $V_{T}$, as well as $T_{I}$, on $\tau$ of the lungs of small rabbits. As a basis for discussing the possible effects of stress relaxation and a distribution of $\tau$ on the measured $\tau$, we calculated the compliance of the respiratory system by three different methods. We measured $\mathrm{C}_{\mathrm{dyn}}, \mathrm{C}_{\mathrm{q} s}$, and $\mathrm{C}_{\mathrm{st}}$.

\section{MATERIALS AND METHODS}

Animal preparation. We studied nine 7 -wk-old New Zealand White rabbits weighing between 1100 and $1350 \mathrm{~g}$ (1239 \pm 230 g, mean $\pm S D$ ). We anesthetized each rabbit with ketamine (30 $\mathrm{mg} / \mathrm{kg})$, acepromazine $(1 \mathrm{mg} / \mathrm{kg})$, and rompun $(0.7 \mathrm{mg} / \mathrm{kg})$ and then placed them in the supine position. Using lidocaine as a local anesthetic, we then introduced an endotracheal tube (3.0mm inner diameter) through a tracheostomy and tied sutures around the trachea to prevent air leakage. We mechanically ventilated the rabbits using a time-cycled, pressure-limited infant ventilator (Baby Bird, Bird Corp, Palm Springs, CA). Finally, we cannulated the femoral artery for drug administration and for monitoring arterial blood pressure and blood-gas tensions. We used changes in arterial blood pressure and heart rate as a guide for the subsequent administration of anesthetics during the experiment. Before measurements, we paralyzed each animal with $0.1 \mathrm{mg} / \mathrm{kg}$ of pancuronium bromide.

Equipment. We measured respiratory flow using a Fleisch " 00 " pneumotachometer (OEM Medical, Inc., Richmond, VA) that was placed between the ventilator tubing " $T$ " and the endotracheal tube. For $\mathrm{C}_{\mathrm{qs}}$ measurements, we fabricated a "clamp connector" using a 3-cm length of 3/8-inch inner diameter polyvinylchloride tubing. We connected the clamp connector to a fitting that was designed to house the large $(15-\mathrm{mm})$ end of an endotracheal tube adapter, similar to the ventilator $T$ connector. One end of the clamp connector was attached to the pneumotachometer and the other end was connected to the endotracheal tube adapter. This allowed us to obstruct flow at a position between the pneumotachometer and the rabbit (5). We monitored airway pressure through a luer-lock side port of the endotracheal tube adapter (Respiratory Support Products Inc., Costa Mesa, CA). Forceps were used to pinch the clamp connector and obstruct flow.

We used three 6-inch long pieces of 1/16-inch polyvinyl- 
chloride tubing to couple the pressure transducers to the pneumotachometer and to the endotracheal tube adapter. We used variable reluctance pressure transducer-carrier demodulator systems (MP-45-871, CD 257, Validyne, Inc., Northridge, CA) with the pneumotachometer $\left( \pm 2 \mathrm{~cm} \mathrm{H}_{2} \mathrm{O}\right)$ and for the airway pressure $\left( \pm 50 \mathrm{~cm} \mathrm{H}_{2} \mathrm{O}\right.$ ). We conditioned the pressure signals using a 50 $\mathrm{Hz}$ low-pass filter (Validyne, Inc.) and then fed the signals to a 12-bit resolution analog to digital converter (Model 570, Keithley, Cleveland, OH). The computer (IBM Inc., Boca Raton, FL) sampled the data at 0.010 -s intervals.

Measurement methods. We measured the $\tau$ during mechanical ventilation using methods similar to those described by LeSouef et al. (5); however, we did not obstruct flow to allow pressures to reach equilibrium throughout the lung. This allowed the respiratory units having a short time constant to become more fully charged than the respiratory units having longer time constants; this reasoning assumes that a distribution of $\tau$ exists. We calculated time constants from the passive deflation flow-volume curves (Fig. 1), which we recorded after adjusting the ventilator to each of the nine different settings.

The ranges of $T_{T}$ and $V_{T}$ chosen encompass the ventilator settings and $V_{T}$ most frequently used on infants in our newborn intensive care unit. We increased the inflation time from 0.2 to $0.4 \mathrm{~s}$ and then to $0.6 \mathrm{~s}$ while the tidal volumes were kept constant at 10,15 , and then $20 \mathrm{~mL}$ by adjusting both the peak inflation pressure and the ventilator flow rate. We maintained the positive end-expiratory pressure at $2 \mathrm{~cm} \mathrm{H}_{2} \mathrm{O}$ to reduce the possibility of partial or sequential airway closure during exhalation. When $T_{1}$ was 0.2 and $0.4 \mathrm{~s}$, the ventilator was time cycled rather than pressure limited. Therefore, the airway pressure increased throughout the time of inflation except during the brief period at the very end of inflation when the ventilator cycled for deflation and yet inflation continued briefly as long as the airway pressure was greater than the alveolar pressure. When the $T_{1}$ was $0.6 \mathrm{~s}$, the ventilator was pressure limited and the airway pressure remained constant for a portion of the ventilator inflation cycle. During this time, when the ventilator was pressure limited, the insufflation flow returned to 0 before the ventilator cycled for deflation. Throughout each experiment, we held the ventilator deflation cycle constant for all of the ventilator settings and adjusted them sufficiently long to insure complete deflation of the lung to a relaxation volume at $2 \mathrm{~cm} \mathrm{H}_{2} \mathrm{O}$ pressure.

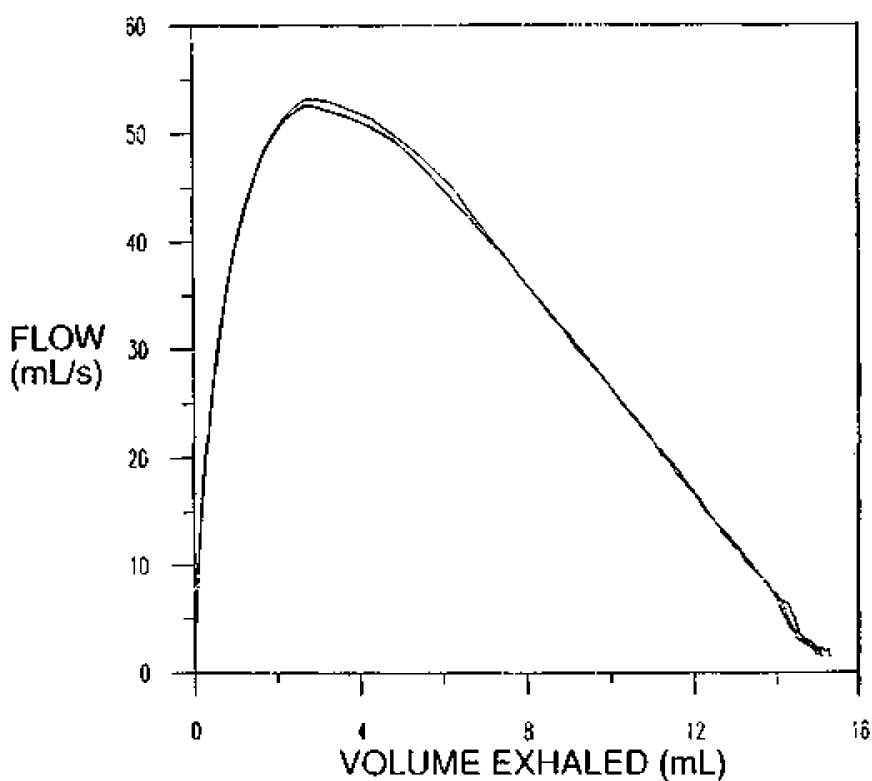

Fig. 1. Passive deflation flow-volume curve of juvenile rabbit receiv. ing mechanical ventilation with a $T_{1}$ of $0.2 \mathrm{~s}$. Three successive breaths are plotted demonstrating good reproducibility and that the plots are linear except in the initial part of deflation where inertial effects dominate.
We calculated time constants using a linear regression with seven points weighted evenly in volume, as opposed to time, from $30-90 \%$ of the deflation volume (6). We determined respiratory system $C_{d y n}$ from the quotient of $V_{T}$ and the difference between the airway pressures at end-inflation and end-deflation. An interpolation subroutine estimated the airway pressures when the flow equaled 0 at end-inflation and end-deflation.

To determine $\mathrm{C}_{\mathrm{q}}$, we clamped the polyvinyl-chloride tubing of the fabricated clamp-connector at, or very near end inflation, disconnected the rabbit from the ventilator while allowing pressures within the lung to reach equilibrium for $2 \mathrm{~s}$, and then released the clamp so that the rabbit exhaled to the atmosphere. We calculated respiratory system $C_{a s}$ from the quotient of the volume exhaled to the atmosphere and the airway pressure measured just before the release of the clamp. We made the $\mathrm{C}_{\mathrm{qs}}$ measurements at volumes of 10,15 , and $20 \mathrm{~mL}$.

We obtained static pressure-volume curves for the respiratory system over a volume range of $30 \mathrm{~mL}$. First, we disconnected the rabbits from the ventilator and allowed them to exhale to atmospheric pressure. Then, using a $50-\mathrm{mL}$ glass syringe connected to the endotracheal tube, we insufflated the lungs with 30 $\mathrm{mL}$ of air and allowed pressures in the lung to equilibrate for 2 s. We then withdrew 5-mL volumes of air and sampled the airway pressure at each lung volume after allowing $2 \mathrm{~s}$ for pressures to equilibrate at each volume. We calculated the chord compliances by dividing the volumes of 10,15 , and $20 \mathrm{~mL}$ by the pressure differences between the airway pressures at these volumes and the airway pressure at $0 \mathrm{~mL}$. We sighed the animals with $30 \mathrm{~cm} \mathrm{H} \mathrm{H}_{2} \mathrm{O}$ airway pressure for 2 -s just before all measurements: dynamic, quasi-static, and static.

Statistics. All data are reported as mean value \pm SEM. We used two-way analysis of variance, with student Newman-Kuels test, to assess differences between the results of the $\tau, C_{d y n}$, and $\mathrm{C}_{\mathrm{q}}$ measurements determined at the different $\mathrm{T}_{1}$ and $\mathrm{V}_{\mathrm{T}}$ settings. A $p<0.05$ was considered significant.

The research protocol was reviewed and approved by the Baylor Animal Protocol Review Committee.

\section{RESULTS}

Increasing $\mathrm{T}_{\mathrm{I}}$ from 0.2 to $0.6 \mathrm{~s}$ while $\mathrm{V}_{\mathrm{T}}$ was held constant at $10 \mathrm{~mL}$ resulted in an increase in $\tau$ of $11 \%(p<0.05)$ (Fig. 2) and an increase in $\mathrm{C}_{\mathrm{dyn}}$ of $10 \%(p<0.05)$ (Table 1). Increasing $V_{T}$ from 10 to $20 \mathrm{~mL}$ while holding $T_{1}$ constant also lead to an average increase in $\tau$ of $11 \%(p<0.05)$, but $C_{d y n}$ decreased $10 \%$ $(p<0.05)$.

Fig. 2. $\tau$ (in s) of rabbits $v s \mathrm{~T}_{1}$ and $\mathrm{V}_{\mathrm{r}}$ (SEM $<0.01 \mathrm{~s}$ for all values). 